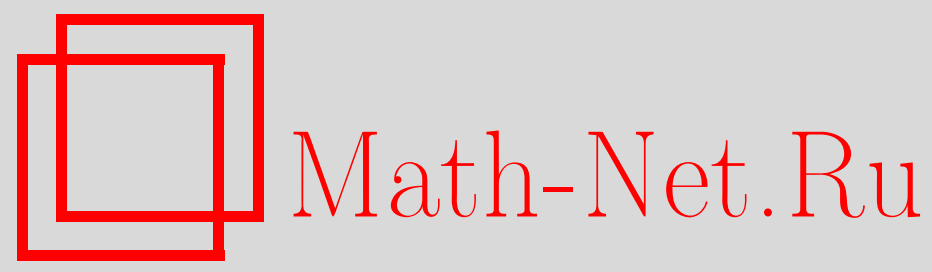

С. С. Марченков, Оператор замыкания с разветвлением по предикату равенства на множестве частичных булевых функций, Дискрет. матем., 2008, том 20, выпуск 3, 80-88

DOI: https://doi.org/10.4213/dm1015

Использование Общероссийского математического портала Math-Net.Ru подразумевает, что вы прочитали и согласны с пользовательским соглашением http://www . mathnet.ru/rus/agreement

Параметры загрузки:

IP: 54.166 .219 .16

26 апреля 2023 г., $17: 59: 22$ 


\title{
Оператор замыкания с разветвлением по предикату равенства на множестве частичных булевых функций
}

\author{
() 2008 г. $\quad$ С. С. Марченков
}

\begin{abstract}
На множестве $P_{2}^{*}$ частичных булевых функций рассматривается оператор замыкания с разветвлением по предикату равенства. Доказывается, что по отношению к этому оператору каждый замкнутый класс из $P_{2}^{*}$ порождается множеством всех своих функций, зависящих не более чем от двух переменных. Определяются все максимальные и субмаксимальные классы в $P_{2}^{*}$. Перечисляются все замкнутые классы, порождаемые одной одноместной или двуместной функцией.

Работа выполнена при поддержке Российского фонда фундаментальных исследований, проект 06-01-00438.
\end{abstract}

\section{1. Введение}

Частичные булевы функции довольно широко изучаются в дискретной математике и математической кибернетике. С функциональной точки зрения частичные булевы функции расположены ближе к функциям трехзначной логики, нежели к обычным булевым функциям. Так, если рассматривать замкнутые (относительно операции суперпозиции) классы частичных булевых функций, то здесь существуют как замкнутые классы, не имеющие базиса [6, 7], так и замкнутые классы со счетным базисом [1]. Из последнего факта следует, что число замкнутых классов частичных булевых функций континуально. Это в известной степени затрудняет изучение порождающих возможностей частичных булевых функций.

Вместе с тем в теории функций многозначной логики известно немало операторов замыкания, которые существенно сильнее оператора суперпозиции и которые при любом $k \geqslant 2$ приводят к конечным классификациям множества функций $k$-значной логики. К таким операторам прежде всего относятся операторы замыкания программного типа $[2,5]$. Спектр этих операторов весьма широк, и далеко не все классификации, отвечающие этим операторам, подробно изучены. Исключение составляет оператор замыкания с разветвлением по предикату равенства [4]. Соответствующие этому оператору замкнутые классы в [4] названы $E$-замкнутыми классами. В [4] показано, что при любом $k \geqslant 2$ имеется лишь конечное число $E$-замкнутых классов $k$-значной логики, содержащих тождественную функцию. Все эти классы допускают альтернативное описание в терминах однородных функций и операции суперпозиции.

В настоящей статье мы исследуем действие оператора замыкания с разветвлением по предикату равенства ( $E$-оператора) на множестве $P_{2}^{*}$ частичных булевых функций. До- 
казывается, что каждый из $E$-замкнутых классов в $P_{2}^{*} E$-порождается множеством всех своих функций, зависящих не более чем от двух переменных (теорема 1). В терминах $E$-предполных классов устанавливается критерий $E$-полноты в классе $P_{2}^{*}$ (теорема 2 ), а также аналогичные критерии в каждом из $E$-предполных классов. В заключительном параграфе перечисляются все $E$-замкнутые классы, которые $E$-порождаются одной одноместной или двуместной функцией.

\section{2. Основные понятия}

Пусть $E_{2}=\{0,1\}, P_{2}-$ множество всех функций на $E_{2}$ (множество булевых функций), $P_{2}^{*}-$ множество всех частичных функций на $E_{2}$ (множество частичных булевых функций). Если $f\left(x_{1}, \ldots, x_{n}\right) \in P_{2}^{*},\left(a_{1}, \ldots, a_{n}\right) \in E_{2}^{n}$ и функция $f$ не определена на наборе $\left(a_{1}, \ldots, a_{n}\right)$, то этот факт будем записывать в виде $f\left(a_{1}, \ldots, a_{n}\right)=*$. Символом $*$ обозначаем также нигде не определенную функцию (от любого числа переменных). Одноместную функцию $f$ из $P_{2}^{*}$ будем иногда записывать в виде вектора $(f(0) f(1))$. Например, вектором $(0 *)$ обозначаем такую функцию $f(x)$, что $f(0)=0$ и $f(1)=*$. Некоторые двуместные функции $f(x, y)$ будем задавать в виде $2 \times 2$ матрицы, у которой в $i$-й строке и $j$-м столбце стоит значение $f(i-1, j-1), 1 \leqslant i, j \leqslant 2$.

Произвольную функцию $f\left(x_{1}, \ldots, x_{n}\right)$ рассматриваем также как функцию от любого большего числа переменных $x_{1}, \ldots, x_{n}, x_{n+1}, \ldots, x_{n+m}$, считая переменные $x_{n+1}, \ldots, x_{n+m}$ несущественными для функции $f$. Так же, как для множества булевых функций [8], с использованием понятия формулы (терма) над множеством функций определяем на множестве $P_{2}^{*}$ операцию суперпозиции. При этом дополнительно, если

$$
f\left(x_{1}, \ldots, x_{n}\right)=g\left(g_{1}\left(x_{1}^{1}, \ldots, x_{k_{1}}^{1}\right), \ldots, g_{m}\left(x_{1}^{m}, \ldots, x_{k_{m}}^{m}\right)\right),
$$

где $\left\{x_{1}^{1}, \ldots, x_{k_{1}}^{1}, \ldots, x_{1}^{m}, \ldots, x_{k_{m}}^{m}\right\} \subseteq\left\{x_{1}, \ldots, x_{n}\right\}$ и значение хотя бы одной из функций $g_{1}, \ldots, g_{m}$ на наборе $\tilde{a}=\left(a_{1}, \ldots, a_{n}\right)$ не определено, либо не определено значение функции $g$ на наборе $\left(g_{1}(\tilde{a}), \ldots, g_{m}(\tilde{a})\right)$, то и значение $f(\tilde{a})$ не определено. Отметим еще, что в суперпозиции (1) некоторые из функций $g_{1}, \ldots, g_{m}$ могут являться тождественными функциями, то есть быть равными значениям какой-либо из своих переменных. В этом случае в суперпозиции (1) оставляем лишь соответствующую переменную.

Пусть $Q \subseteq P_{2}^{*}$. Замыканием $Q$ называется множество всех функций из $P_{2}^{*}$, которые можно получить из функций множества $Q$ с помощью операций введения несущественных переменных и суперпозиции, замыкание множества $Q$ обозначаем $[Q]$. Множество $Q$ называется замкнутым, если $Q=[Q]$. Замкнутые множества называем также замкнутыми классами.

Пусть $T_{0}, T_{1}, S, C_{0}, C_{1}$ обозначают замкнутые классы функций из $P_{2}$, которые соответственно сохраняют 0 , сохраняют 1 , самодвойственны, равны константе 0 , равны константе 1 (см. [9, 3]). Положим далее $T_{01}=T_{0} \cap T_{1}, S_{01}=S \cap T_{0}$.

Пусть $g_{1}, g_{2} \in P_{2}^{*}$. Говорим, что функция $f\left(x_{1}, \ldots, x_{n}\right)$ получается из функций $g_{1}\left(x_{1}, \ldots, x_{n}\right), g_{2}\left(x_{1}, \ldots, x_{n}\right)$ с помощью операции разветвления по предикату равенства, если для некоторых $i, j \in\{1,2, \ldots, n\}$ выполняется соотношение

$$
f\left(x_{1}, \ldots, x_{n}\right)= \begin{cases}g_{1}\left(x_{1}, \ldots, x_{n}\right), & \text { если } x_{i}=x_{j}, \\ g_{2}\left(x_{1}, \ldots, x_{n}\right) & \text { в противном случае. }\end{cases}
$$

$E$-замыкание множества $Q \subseteq P_{2}^{*}$ определяем как множество всех функций из $P_{2}^{*}$, которые можно получить из функций множества $Q$ с помощью операций введения несущественных переменных, суперпозиции и разветвления по предикату равенства. 
$E$-замыкание множества $Q$ обозначаем $[Q]_{E}$. Множество функций, которое совпадает со своим $E$-замыканием, называем $E$-замкнутым классом. Говорим, что множество $R \subseteq Q$ $E$-порождает $E$-замкнутый класс $Q$ (или $E$-полно в классе $Q$ ), если $[R]_{E}=Q$.

Обозначим через $T_{0}^{*}$ множество всех функций из $P_{2}^{*}$, которые на нулевом наборе принимают значение 0 или $*$. Аналогично определяем множество $T_{1}^{*}$. Пусть $S^{*}$ есть множество всех функций из $P_{2}^{*}$, которые на любой паре противоположных наборов принимают значения, отличные от $(0,0)$ и $(1,1)$. Обозначим через $I^{*}$ множество всех функций из $P_{2}^{*}$, которые переводят нулевой и единичный наборы в одну из пар $(0,1),(0, *),(1, *)$, $(*, 0),(*, 1),(*, *)$.

Нетрудно убедиться в том, что классы $P_{2}, T_{0}^{*}, T_{1}^{*}, S^{*}, I^{*}$ являются $E$-замкнутыми. При этом при рассмотрении операции (2) разветвления по предикату равенства следует обратить внимание на то, что нулевой и единичный наборы попадают в первую часть схемы (2), а пары противоположных наборов одновременно входят либо в первую, либо во вторую части схемы (2). Отметим также $E$-замкнутость классов $T_{0}, T_{1}, S, T_{01}, S_{01}$, которая установлена в [4], и очевидную $E$-замкнутость классов $C_{0}$ и $C_{1}$.

\section{3. Предварительные результаты}

В [4] доказано, что для любого множества $Q \subseteq P_{2}$, содержащего тождественную функцию $x, E$-замыкание множества $Q$ совпадает с $\left[Q \cup S_{01}\right]$. Отсюда и из известных фактов о строении решетки замкнутых классов булевых функций $[9,3]$ следует, что система функций $\{0,1\} E$-полна в классе $P_{2}$, система $\{0, x\} E$-полна в классе $T_{0}$, система $\{1, x\}$ $E$-полна в классе $T_{1}$, функция $\bar{x}$ образует $E$-полную систему в классе $S$, функция $x y$ (а также функция $x \vee y$ ) образует $E$-полную систему в классе $T_{01}$, функция $x$ образует $E$-полную систему в классе $S_{01}$.

Очевидно также, что классы $C_{0}, C_{1} E$-порождаются соответственно константами 0 и 1 . Поскольку $C_{0}, C_{1}$ являются единственными замкнутыми классами в $P_{2}$, которые не содержат ни функции $x$, ни одновременно двух констант $[9,3]$, приходим к выводу, что в классе $P_{2}$ помимо самого класса $P_{2}$ имеется только $7 E$-замкнутых классов: $T_{0}, T_{1}, S$, $T_{01}, S_{01}, C_{0}, C_{1}$.

Используем отмеченные выше результаты о $E$-порождающих системах в классах $P_{2}$, $T_{0}, T_{1}, S, T_{01}$ для построения $E$-полных систем в классах $P_{2}^{*}, T_{0}^{*}, T_{1}^{*}, S^{*}, I^{*}$.

Лемма 1. Система $\{0,1, *\}$ E-полна в классе $P_{2}^{*}$.

Доказательство. Используя тождественную функцию из класса $P_{2}=[\{0,1\}]_{E}$, определяем с помощью операции разветвления по предикату равенства функцию $g$, полагая

$$
g(x, y, z)= \begin{cases}z, & \text { если } x=y, \\ * & \text { в противном случае. }\end{cases}
$$

Пусть $f(\tilde{x})$ - произвольная функция из $P_{2}^{*}, f^{\prime}(\tilde{x})-$ ее доопределения в классе $P_{2}$, $f_{1}(\tilde{x}), f_{2}(\tilde{x})$ - такие функции из $P_{2}$, что

$$
\left(f_{1}(\tilde{x})=f_{2}(\tilde{x})\right) \equiv(f(\tilde{x}) \text { определено }) .
$$

Тогда $\left.f(\tilde{x})=g\left(f_{1}(\tilde{x})\right), f_{2}(\tilde{x}), f^{\prime}(\tilde{x})\right)$. Лемма доказана.

Лемма 2. Система $\{0, x, *\}$-полна в классе $T_{0}^{*}$, система $\{1, x, *\} E$-полна в классе $T_{1}^{*}$. 
Доказательство. Рассмотрим только класс $T_{0}^{*}$. Так же, как и в лемме 1 , из тождественной функции и функции * получаем функцию $g$. Пусть $f\left(x_{1}, \ldots, x_{n}\right) \in T_{0}^{*}, f^{\prime}(\tilde{x})-$ доопределение функции $f$ в классе $T_{0}, f(\tilde{x}), f_{2}(\tilde{x})$ - такие функции из класса $T_{0}$, что на множестве $E_{2}^{n} \backslash\{\tilde{0}\}$ справедлива эквивалентность (3). Положим

$$
f_{3}(\tilde{x})=g\left(f_{1}(\tilde{x}), f\left(2(\tilde{x}), f^{\prime}(\tilde{x})\right) .\right.
$$

Тогда $f_{3}(\tilde{0})=0$ и на множестве $E_{2}^{n} \backslash\{\tilde{0}\}$ функция $f_{3}$ совпадает с функцией $f$. Если $f_{3}$ отлична от функции $f$, то должно выполняться равенство $f(\tilde{x})=*$. В этом случае по аналогии с функцией $f_{3}$ определим функцию $f_{4}$, которая равна 0 на наборе $\tilde{0}$ и отлична от функции $f_{3}$ на любом наборе из $E_{2}^{n} \backslash\{\tilde{0}\}$, на котором функция $f_{3}$ определена. Положим

$$
g_{1}(x, y)= \begin{cases}*, & \text { если } x=y, \\ x & \text { в противном случае. }\end{cases}
$$

Тогда $f(\tilde{x})=g_{1}\left(f_{3}(\tilde{x}), f_{4}(\tilde{x})\right)$. Лемма доказана.

Лемма 3. Система $\{\bar{x},(0 *)\}$ E-полна в классе $S^{*}$.

Доказательство. Понятно, что суперпозициями функций $\bar{x},(0 *)$ можно получить любую одноместную функцию из класса $S^{*}$.

Пусть $f(\tilde{x})$ - произвольная функция из класса $S^{*}, f^{\prime}(\tilde{x})$ - ее доопределение в классе $S$. Если функция $f$ отлична от функции $f^{\prime}$ (напомним, что функция $\bar{x}$ образует $E$-базис класса $S$ ), то пусть, например, функция $f$ не определена на наборе $\tilde{a}$. Выберем в классе $S$ такие функции $f_{1}(\tilde{x}), f_{2}(\tilde{x})$, что неравенство $f_{1}(\tilde{x}) \neq f_{2}(\tilde{x})$ выполняется только для набора $\tilde{a}$ и противоположного ему набора $\tilde{a}^{\prime}$. Пусть далее $g(x)$ - такая функция из $S^{*}$, что $g\left(f_{1}(\tilde{a})\right)=f(\tilde{a}), g\left(f_{1}\left(\tilde{a}^{\prime}\right)\right)=f\left(\tilde{a}^{\prime}\right)$. Положим

$$
\begin{aligned}
g_{1}(x, y, z) & = \begin{cases}z, & \text { если } x=y, \\
g(x) & \text { в противном случае, }\end{cases} \\
f_{3}(\tilde{x}) & =g_{1}\left(f_{1}(\tilde{x}), f_{2}(\tilde{x}), f^{\prime}(\tilde{x})\right) .
\end{aligned}
$$

Тогда функция $f_{3}$, будет совпадать с функцией $f$ на наборах $\tilde{a}, \tilde{a}^{\prime}$ и всех наборах $\tilde{x}$, на которых значение $f$ определено.

Пусть теперь $f_{3}^{1}(\tilde{x}), \ldots, f_{3}^{t}(\tilde{x})-$ все функции типа $f_{3}(\tilde{x})$, построенные для всех указанных выше наборов $\tilde{a}, h\left(x_{1}, \ldots, x_{t}\right)$ - функция из $S$, сохраняющая 0. Тогда $f(\tilde{x})=h\left(f_{3}^{1}(\tilde{x}), \ldots, f_{3}^{t}(\tilde{x})\right)$. Лемма доказана.

Лемма 4. Система $\{x y,(1 *),(* 0)\}$ E-полна в классе $I^{*}$.

Доказательство. Суперпозициями функций $(1 *),(* 0)$ получаем еще три не всюду определенные функции класса $I^{*}$, а именно, $(0 *),(* 1), *$.

Пусть $f\left(x_{1}, \ldots, x_{n}\right)$ - произвольная функция из класса $I^{*}$. Выберем в классе $T_{01}$, который $E$-порождается функцией $x y$, такие функции $f^{\prime}(\tilde{x}), f_{1}(\tilde{x}), f_{2}(\tilde{x})$, что на множестве $E_{2}^{n} \backslash\{\tilde{0}, \tilde{1}\}$ функция $f^{\prime}$ является доопределением функции $f$, а функции $f_{1}, f_{2}$ на этом множестве удовлетворяют эквивалентности (3). Положим

$$
f_{3}(\tilde{x})=g\left(f_{1}(\tilde{x}), f_{2}(\tilde{x}), f^{\prime}(\tilde{x})\right),
$$

где $g$ - функция из доказательства леммы 1 . Тогда $f_{3}(\tilde{0})=0, f_{3}(\tilde{1})=1$ и на множестве $E_{2}^{n} \backslash\{\tilde{0}, \tilde{1}\}$ функция $f_{3}$ совпадает с функцией $f$. Если функция $f_{3}$ не равна функции $f$, то 
подобно функции $f_{3}$ определяем функцию $f_{4}(\tilde{x})$, которая отличается от функции $f_{3}(\tilde{x})$ на каждом наборе $\tilde{x}$ из множества $E_{2}^{n} \backslash\{\tilde{0}, \tilde{x}\}$, на котором функция $f_{3}$ определена. Пусть $h(x)$ - такая функция из класса $I^{*}$, что $h(0)=f(\tilde{0}), h(1)=f(\tilde{1})$. Положим

$$
g_{1}(x, y)= \begin{cases}h(x), & \text { если } x=y \\ x & \text { в противном случае. }\end{cases}
$$

Тогда $f(\tilde{x})=g_{1}\left(f_{3}(\tilde{x}), f_{4}(\tilde{x})\right)$. Лемма доказана.

Следующая теорема показывает, в частности, что число $E$-замкнутых классов в $P_{2}^{*}$ конечно.

Теорема 1. Любой E-замкнутый класс функций из $P_{2}^{*}$ E-порождается множеством всех своих функиий, зависящих не более чем от двух переменных.

Доказательство. Пусть $f\left(x_{1}, \ldots, x_{n}\right) \in P_{2}^{*}$ и $n \geqslant 3$. Покажем, что функцию $f$ можно получить из функций

$$
\begin{aligned}
f_{1}\left(x_{1}, x_{3}, \ldots, x_{n}\right) & =f\left(x_{1}, x_{1}, x_{3}, \ldots, x_{n}\right), \\
f_{2}\left(x_{1}, x_{2}, x_{4}, \ldots, x_{n}\right) & =f\left(x_{1}, x_{2}, x_{1}, x_{4}, \ldots, x_{n}\right), \\
f_{3}\left(x_{1}, x_{2}, x_{4}, \ldots, x_{n}\right) & =f\left(x_{1}, x_{2}, x_{2}, x_{4}, \ldots, x_{n}\right)
\end{aligned}
$$

с помощью операции разветвления по предикату равенства. Поскольку функции $f_{1}, f_{2}$, $f_{3}$ зависят от $n-1$ переменных, отсюда следует утверждение теоремы. В самом деле, положим

$$
f^{\prime}\left(x_{1}, \ldots, x_{n}\right)= \begin{cases}f_{2}\left(x_{1}, x_{2}, x_{4}, \ldots, v_{n}\right), & \text { если } x_{1}=x_{3}, \\ f_{3}\left(x_{1}, x_{2}, x_{4}, \ldots, x_{n}\right) & \text { в противном случае. }\end{cases}
$$

Заметим, что функция $f^{\prime}$ совпадает с функцией $f$ на любом наборе $\left(a_{1}, a_{2}, \ldots, a_{n}\right)$, где $a_{1} \neq a_{2}$. Поэтому функция $f$ определяется соотношениями

$$
f\left(x_{1}, \ldots, x_{n}\right)= \begin{cases}f_{1}\left(x_{1}, x_{3}, \ldots, x_{n}\right), & \text { если } x_{1}=x_{2}, \\ f^{\prime}\left(x_{1}, \ldots, x_{n}\right) & \text { в противном случае. }\end{cases}
$$

Теорема доказана.

\section{4. Максимальные и субмаксимальные классы}

Пусть $Q, R-E$-замкнутые классы и $R \subset Q$. Класс $R$ называется $E$-максимальным ( $E$-предполным) в классе $Q$, если не существует такого $E$-замкнутого класса $V$, что $R \subseteq V \subseteq Q$ и $V$ отличен от классов $R, Q$.

Следующая теорема показывает, что классы $P_{2}, T_{0}^{*}, T_{1}^{*}, S^{*}, I^{*}$ (которые, как нетрудно видеть, целиком не содержатся друг в друге) являются единственными $E$-предполными в $P_{2}^{*}$ классами.

Теорема 2 (критерий $E$-полноты в классе $P_{2}^{*}$ ). Система функций из $P_{2}^{*} E$-полна в классе $P_{2}^{*}$ тогда и только тогда, когда она иеликом не содержится ни в одном из классов $P_{2}, T_{0}^{*}, T_{1}^{*}, S^{*}, I^{*}$. 
Доказательство. Необходимость условия теоремы следует из того, что классы $P_{2}, T_{0}^{*}$, $T_{1}^{*}, S^{*}, I^{*} E$-замкнуты и отличны от класса $P_{2}^{*}$.

Установим достаточность условия теоремы. Пусть $f_{2}, \ldots, f_{5}$ - функции, которые не входят соответственно в классы $P_{2}, T_{0}^{*}, T_{1}^{*}, S^{*}, I^{*}$. Покажем, что система $\left\{f_{2}, \ldots, f_{5}\right\}$ $E$-полна в классе $P_{2}^{*}$.

Из определения классов $P_{2}, T_{0}^{*}, T_{1}^{*}, S^{*}, I^{*}$ следует, что отождествлением переменных из функций $f_{2}, \ldots, f_{5}$ можно получить такие функции $g_{1}(x, y), g_{2}(x), g_{3}(x), g_{4}(x, y)$, $g_{5}(x)$, что

$$
g_{1}(0,1)=*, \quad g_{2}(0)=1, \quad g_{3}(1)=0, \quad g_{4}(0,1)=g_{4}(1,0) \neq *, \quad g_{5}(x) \in\{0,1, \bar{x}\} .
$$

Если $g_{5}$ - константа, то другую константу получаем подстановкой функции $g_{5}$ в функции $g_{2}$ или $g_{3}$. Если $g_{5}(x)=\bar{x}$, то константой является функция $g_{4}\left(x, g_{5}(x)\right)$. Имея обе константы, получаем из функции $g_{1}$ функцию $*$. Далее применяем лемму 1 . Теорема доказана.

Наша дальнейшая цель - найти все субмаксимальные классы в $P_{2}^{*}$, то есть все $E$-замкнутые классы, которые являются максимальными хотя бы в одном из классов $P_{2}, T_{0}^{*}, T_{1}^{*}, S^{*}, I^{*}$. Для класса $P_{2}$ мы фактически сделали это выше, перечислив все $E$-замкнутые классы из $P_{2}$ : все $E$-замкнутые классы в $P_{2}$ суть $T_{0}, T_{1}, S$. Для остальных классов $T_{0}^{*}, T_{1}^{*}, S^{*}, I^{*}$ это будет сделано в теоремах 3-5.

Пусть $T_{0,0}^{*}-$ множество всех функций из $P_{2}^{*}$, сохраняющих константу $0, T_{1,1}^{*}-$ множество всех функций из $P_{2}^{*}$, сохраняющих константу 1 . Обозначим через $T_{0,1}^{*}$ множество всех функций из класса $T_{0}^{*}$, которые не принимают значение 1. Двойственным образом определим класс $T_{1,0}^{*}$. Нетрудно установить $E$-замкнутость классов $T_{0,0}^{*}, T_{1,1}^{*}, T_{0,1}^{*}, T_{1,0}^{*}$.

Теорема 3 (критерий $E$-полноты в классе $T_{0}^{*}$ ). Система функций из $T_{0}^{*} E$-полна в классе $T_{0}^{*}$ тогда и только тогда, когда она целиком не содержится ни в одном из классов $T_{0,0}^{*}$, $T_{0,1}^{*}, T_{0}^{*} \cap I^{*}$.

Доказательство. Необходимость условия теоремы следует из $E$-замкнутости классов $T_{0,0}^{*}, T_{0,1}^{*}, T_{0}^{*} \cap I^{*}$ и несовпадения их с классом $T_{0}^{*}$.

Докажем достаточность условия теоремы. Пусть $f_{1}, f_{2}, f_{3}$ - функции класса $T_{0}^{*}$, которые не принадлежат соответственно классам $T_{0,0}^{*}, T_{0,1}^{*}, T_{0}^{*} \cap I^{*}$. Из определения этих классов следует, что отождествлением переменных из функций $f_{1}, f_{2}, f_{3}$ можно получить такие функции $g_{1}(x), g_{2}(x, y), g_{3}(x)$, что $g_{1}(0)=*, g_{2}(0,1)=1, g_{3}(x)=0$. Подстановка функции $g_{3}$ в функцию $g_{1}$ дает $*$, а суперпозиция $g_{2}\left(g_{3}(x), x\right)$ - одну из функций $x,(* 1)$. В случае функции $x$ в силу леммы 2 получаем $E$-полную в классе $T_{0}^{*}$ систему $\{0, x, *\}$. Если же имеется функция $(* 1)$, то далее полагаем

$$
g_{4}(x, y)= \begin{cases}0, & \text { если } x=y, \\ (* 1)(x) & \text { в противном случае }\end{cases}
$$

Теперь получаем функцию $g_{4}(x, 0)=x$. Теорема доказана.

Аналогично теореме 3 устанавливается критерий $E$-полноты в классе $T_{1}^{*}$.

Пусть $S^{* *}$-множество всех функций из $P_{2}^{*}$, которые на любой паре противоположных наборов принимают лишь значения $(0,1),(1,0)$ или $(* *)$. Легко видеть, что $S^{* *}-$ $E$-замкнутый класс, целиком лежащий в $S^{*}$. 
Теорема 4 (критерий полноты в классе $S^{*}$ ). Система функциий из $S^{*}$ E-полна в классе $S^{*}$ тогда и только тогда, когда она иеликом не содержстся ни в одном из классов $S^{*} \cap I^{*}, S^{* *}$.

Доказательство. Докажем лишь достаточность условий. Пусть $f_{1} \in S^{*} \backslash I^{*}$, $f_{2} \in S^{*} \backslash S^{* *}$. Используя определения классов $S^{*}, I^{*}, S^{* *}$, заключаем, что из функций $f_{1}, f_{2}$ отождествлением переменных можно получить такие функции $g_{1}(x), g_{2}(x)$, что $g_{1}(x)=\bar{x}, g_{2}(x) \in\{(a *),(* a)\}$, где $a \neq *$. Суперпозициями функции $\bar{x}$ и любой из функций $(a *),(* a)$ можно определить функцию $(0 *)$. Далее применяем лемму 3. Теорема доказана.

Пусть $I_{01}^{*}-$ множество всех таких функций из $P_{2}^{*}$, которые не определены на нулевом или единичном наборах. Нетрудно убедиться, в том, что $I_{01}^{*}-E$-замкнутый класс, целиком лежащий в классе $I^{*}$.

Теорема 5 (критерий $E$-полноты в классе $\left.I^{*}\right)$. Cистема функиий из $I^{*} E$-полна в классе $I^{*}$ тогда и только тогда, когда она ичеликом не содержится ни в одном из классов $T_{0}^{*} \cap I^{*}, T_{1}^{*} \cap I^{*}, S^{*} \cap I^{*}, I_{01}^{*}$.

Доказательство. Докажем лишь достаточность условий теоремы. Пусть $f_{1}, f_{2}, f_{3}, f_{4}-$ функции из класса $I^{*}$, которые не принадлежат соответственно классам $T_{0}^{*}, T_{1}^{*}, S^{*}, I_{0,1}^{*}$. Используя определения классов $I^{*}, T_{0}^{*}, T_{1}^{*}, S, I_{0,1}^{*}$, заключаем, что отождествлением переменных из функций $f_{1}, f_{2}, f_{3}, f_{4}$ можно получить такие функции $g_{1}(x), g_{2}(x)$, $g_{3}(x, y), g_{4}(x)$, что $g_{1}(x)=(1 *), g_{2}(x)=(* 0), g_{3}(0,1)=g_{3}(1,0)=a \neq *, g_{4}(x)=x$. Поскольку мы имеем функцию $(* 0)$, можно считать, что $a=0$. Далее получаем, что

$$
x y= \begin{cases}x, & \text { если } x=y, \\ g_{3}(x, y) & \text { в противном случае, }\end{cases}
$$

и применяем лемму 4. Теорема доказана.

С использованием теоремы 1 несложно показать, что

- система $\{0, x,(0 *)\} E$-полна в классе $T_{0,0}^{*}$,

- система $\{0,(0 *),(* 0)\} E$-полна в классе $T_{0,1}^{*}$,

- система $\{x y,(0 *),(* 0),(* 1)\} E$-полна в классе $T_{0}^{*} \cap I^{*}$,

- система $\{1, x,(* 1)\} E$-полна в классе $T_{1,1}^{*}$,

- система $\{1,(1 *),(* 1)\} E$-полна в классе $T_{1,0}^{*}$,

- система $\{x y,(0 *),(1 *),(* 1)\} E$-полна в классе $T_{1}^{*} \cap I^{*}$,

- система $\{x,(1 *),(* 0)\} E$-полна в классе $S^{*} \cap I^{*}$,

- система $\{\bar{x}, *\} E$-полна в классе $S^{* *}$,

- система $\left\{\left(\begin{array}{ll}* & 0 \\ 0 & 0\end{array}\right),\left(\begin{array}{ll}1 & 1 \\ 0 & *\end{array}\right)\right\} E$-полна в классе $I_{0,1}^{*}$. 


\section{E-замкнутые классы, $E$-порождаемые одной двуместной функцией}

В этом параграфе мы исследуем нижнюю часть решетки $E$-замкнутых классов из $P_{2}^{*}$. Именно, будут перечислены все $E$-замкнутые классы, которые $E$-порождаются одной одноместной или двуместной функцией. В некоторых случаях мы полностью охарактеризуем $E$-замкнутый класс, $E$-порождаемый функцией, в других случаях, имея в виду теорему 1, перечислим лишь все одноместные и двуместные функции из этого класса.

Начнем с одноместных функций. Как отмечалось выше, функции $0,1, x, \bar{x} E$-порождают соответственно классы $C_{0}, C_{1}, S_{01}, S$. Функция $*$, очевидно, $E$-порождает класс, который состоит только из нигде не определенных функций. Двойственные друг другу функции $(0 *)$ и $(* 1) E$-порождают классы, которые с точностью до несущественных переменных состоят соответственно из функций $(0 *)(* 1)$. Функция $(1 *) E$-порождает класс, все одноместные и двуместные функции которого суть $\left(\begin{array}{l}1 \\ *\end{array}\right), *,\left(\begin{array}{ll}* & 1 \\ * & *\end{array}\right),\left(\begin{array}{ll}* & 1 \\ * & *\end{array}\right),\left(\begin{array}{ll}* & * \\ 1 & *\end{array}\right)$.

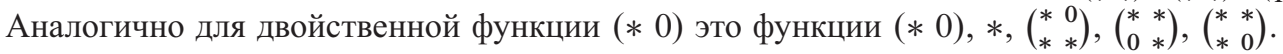

Очевидно, что все (непустые) минимальные классы в решетке $E$-замкнутых классов из $P_{2}^{*} E$-порождаются одноместными функциями. Анализ перечисленных выше $E$-замкнутых классов показывает, что в $P_{2}^{*}$ имеется ровно 6 минимальных $E$-замкнутых классов, которые $E$-порождаются функциями $0,1, x, *,(0 *),(* 1)$.

Перейдем собственно к двуместным функциям. Как отмечалось выше, любая двуместная функция из $P_{2}$, существенно зависящая от обеих переменных, $E$-порождает один из классов $P_{2}, T_{0}, T_{1}, T_{01}$. Например, каждая из функций $x \oplus y, x \bar{y} E$-порождает класс $T_{0}$.

Рассмотрим далее двуместные не всюду определенные функции. Чтобы уменьшить перебор, подобные функции будем рассматривать с точностью до двойственности и перестановки переменных. Примем следующее соглашение: ниже в строках, состоящих из одноместных и двуместных функций, первой будет стоять исходная функция от двух переменных, затем все остальные одноместные и двуместные функции, которые она $E$-порождает. Несущественные переменные в функциях опускаем.

Вначале приведем две функции, которые определены только на одном наборе (остальные функции этого типа либо двойственны данным функциям, либо получаются из них перестановкой переменных, либо $E$-порождают классы, уже рассмотренные выше):

$$
\begin{aligned}
& \left(\begin{array}{ll}
0 & * \\
* & *
\end{array}\right),\left(\begin{array}{ll}
0 & *
\end{array}\right), \\
& \left(\begin{array}{ll}
* & 0 \\
* & *
\end{array}\right), *,\left(\begin{array}{ll}
* & * \\
0 & *
\end{array}\right) .
\end{aligned}
$$

Далее рассмотрим функции, которые не определены ровно на двух наборах:

$$
\begin{aligned}
& \left(\begin{array}{ll}
0 & 1 \\
* & *
\end{array}\right),\left(\begin{array}{ll}
0 & *
\end{array}\right),\left(\begin{array}{ll}
0 & * \\
1 & *
\end{array}\right),\left(\begin{array}{ll}
0 & * \\
* & *
\end{array}\right), \\
& \left(\begin{array}{ll}
0 & * \\
* & 0
\end{array}\right), 0,\left(\begin{array}{ll}
0 & *
\end{array}\right),\left(\begin{array}{ll}
0 & 0 \\
0 & *
\end{array}\right),\left(\begin{array}{ll}
0 & 0 \\
* & 0
\end{array}\right),\left(\begin{array}{ll}
0 & * \\
0 & 0
\end{array}\right),\left(\begin{array}{ll}
0 & * \\
* & *
\end{array}\right), \\
& \left(\begin{array}{ll}
0 & * \\
* & 1
\end{array}\right),\left(\begin{array}{ll}
0 & 1
\end{array}\right), \\
& \left(\begin{array}{ll}
1 & 0 \\
* & *
\end{array}\right),\left(\left(\begin{array}{ll}
0 & *
\end{array}\right),\left(\begin{array}{ll}
1 & *
\end{array}\right), *,\left(\begin{array}{ll}
0 & 1 \\
* & *
\end{array}\right),\left(\begin{array}{ll}
0 & * \\
1 & *
\end{array}\right),\left(\begin{array}{ll}
0 & * \\
* & *
\end{array}\right),\left(\begin{array}{ll}
1 & * \\
0 & *
\end{array}\right),\left(\begin{array}{ll}
1 & * \\
* & *
\end{array}\right),\left(\begin{array}{ll}
* & 0 \\
* & *
\end{array}\right),\left(\begin{array}{ll}
* & * \\
0 & *
\end{array}\right),\left(\begin{array}{ll}
* & 1 \\
* & *
\end{array}\right),\left(\begin{array}{ll}
* & * \\
1 & *
\end{array}\right),\right. \\
& \left(\begin{array}{ll}
1 & * \\
* & 0
\end{array}\right),\left(\begin{array}{ll}
0 & 1
\end{array}\right),\left(\begin{array}{ll}
1 & 0
\end{array}\right),\left(\begin{array}{ll}
0 & * \\
* & 1
\end{array}\right), \\
& \left(\begin{array}{ll}
* & 0 \\
0 & *
\end{array}\right), *, \\
& \left(\begin{array}{ll}
* & 0 \\
1 & *
\end{array}\right), *,\left(\begin{array}{ll}
* & 1 \\
0 & *
\end{array}\right) .
\end{aligned}
$$


В заключение рассмотрим функции, которые не определены только на одном наборе:

$\left(\begin{array}{ll}0 & 0 \\ 0 & *\end{array}\right),(0 *),\left(\begin{array}{ll}0 & * \\ * & *\end{array}\right)$,

$\left(\begin{array}{ll}0 & 0 \\ 1 & *\end{array}\right),\left(\begin{array}{ll}0 & *\end{array}\right),\left(\begin{array}{ll}0 & 1 \\ 0 & *\end{array}\right),\left(\begin{array}{ll}0 & * \\ * & *\end{array}\right)$,

$\left(\begin{array}{ll}0 & 1 \\ 1 & *\end{array}\right),(0 *),\left(\begin{array}{ll}0 & * \\ * & *\end{array}\right)$,

$\left(\begin{array}{ll}1 & 0 \\ 0 & *\end{array}\right)$, все функции $f(x, y)$, которые удовлетворяют условию $f(1,1)=*$,

$\left(\begin{array}{ll}1 & 0 \\ 1 & *\end{array}\right)$, все функции $f(x, y)$, которые удовлетворяют условиям $f(1,1)=*$ и $(f(0,1), f(1,0)) \notin\{(0,0),(1,1)\}$,

$\left(\begin{array}{ll}1 & 1 \\ 1 & *\end{array}\right),(1 \quad *), *,\left(\begin{array}{ll}1 & * \\ * & *\end{array}\right),\left(\begin{array}{ll}* & 1 \\ 1 & *\end{array}\right),\left(\begin{array}{ll}* & 1 \\ * & *\end{array}\right),\left(\begin{array}{ll}* & * \\ 1 & *\end{array}\right)$,

$\left(\begin{array}{ll}0 & 0 \\ * & 0\end{array}\right), 0,(0 *),\left(\begin{array}{ll}0 & 0 \\ 0 & *\end{array}\right),\left(\begin{array}{ll}0 & * \\ 0 & 0\end{array}\right),\left(\begin{array}{ll}0 & * \\ * & 0\end{array}\right),\left(\begin{array}{ll}0 & * \\ * & *\end{array}\right)$,

$\left(\begin{array}{ll}0 & 0 \\ * & 1\end{array}\right),\left(\begin{array}{ll}0 & 1\end{array}\right),\left(\begin{array}{ll}0 & * \\ 0 & 1\end{array}\right),\left(\begin{array}{ll}0 & * \\ * & 1\end{array}\right)$,

$\left(\begin{array}{ll}0 & 1 \\ * & 1\end{array}\right)$, все функции $f(x, y)$, которые сохраняют $0\left(E\right.$-базис класса $\left.T_{0,0}^{*}\right)$

$\left(\begin{array}{ll}0 & 1 \\ * & 1\end{array}\right),\left(\begin{array}{ll}0 & 1\end{array}\right),\left(\begin{array}{ll}0 & * \\ 1 & 1\end{array}\right),\left(\begin{array}{ll}0 & * \\ * & 1\end{array}\right)$,

$\left(\begin{array}{ll}1 & 0 \\ * & 0\end{array}\right)$, все функции $f(x, y)$ из класса $S^{*}(E$-базис класса $S *)$.

\section{Список литературы}

1. Алексеев В. Б., Вороненко А. А., О некоторых замкнутых классах в частичной двузначной логике. Дискретная математика (1994) 6, №4, 58-79.

2. Голунков Ю. В., Критерий полноты системы операций в операторных алгоритмах, реализующих функции $k$-значной логики. Вероятностные методы и кибернетика (1980) 17, 23-34.

3. Марченков С. С., Замкнутые классы булевых функиий. Физматлит, Москва, 2000.

4. Марченков С. С., Операторы замыкания с разветвлением по предикату. Вестник МГУ, Сер. 1: Математика и механика (2003) №6, 37-39.

5. Тайманов В. А., О функциональных системах $k$-значной логики с операциями замыкания программного типа. Докл. АН СССР (1983) 268, №6, 1307-1310.

6. Фрейвалд Р. В., Критерий полноты для частичных функций алгебры логики и многозначных логик. Докл. АН СССР (1966) 167, №6, 1249-1250.

7. Фрейвалд Р. В., Функциональная полнота для не всюду определенных функций алгебры логики. Дискретный анализ (1966) 8, 55-68.

8. Яблонский С. В., Введение в дискретную математику. Наука, Москва, 1986.

9. Яблонский С. В., Гаврилов Г. П., Кудрявцев В. Б., Функции алгебры логики и классы Поста. Наука, Москва, 1966.

Статья поступила 3.01.2006. 